\section{Use of a DNA Sequencing Gel Apparatus for Analy- sis of Polypeptides}

BioTechnsiques 21:805-808 (November 1996)

Polyacrylamide gel electrophoresis (PAGE) is a standard procedure for identifying and analyzing polypeptides (1). Samples are prepared from cell lysates, subjected to electrophoresis and stained with silver nitrate or Coomassie ${ }^{\circledR}$ Brilliant Blue (CBB) allowing direct visualization of bands within gels $(1,5)$. Samples can also be radiolabeled, for example with $\left[{ }^{35}\right.$ S $]$ methionine, and after electrophoresis, the resulting gels are dried on chromatography paper and autoradiographed (3). A wide variety of electrophoresis chambers are available for producing gels for these types of analyses. We describe here a procedure, which allows one to use a nucleic acid sequencing gel apparatus instead of the conventional equipment. The main advantage of this method is that it enables the use of one apparatus for both DNA sequencing and polypeptide work.

To prepare a sequencing apparatus before sealing, we used a Sequi-Gen ${ }^{\mathrm{TM}}$ cell $(21-\times 50-\mathrm{cm})($ Bio-Rad, Hercules, CA, USA) for carrying out this procedure. The clamp set, which is used to hold the glass plates together, should be in good condition. Warped or uneven clamps cause leakage at the sides during gel pouring. Plates and spacers are washed normally and rinsed with $70 \%$ ethanol. No treatment is required to selectively bind the acrylamide gel to the outer glass plate. Four 0.4-mm spacers are required. These are doubled together along each side of the apparatus, giving a gap of $0.8 \mathrm{~mm}$, once the assembly is clamped together. Thinner gels $(0.4$ $\mathrm{mm}$ ) are extremely difficult to pour, and they break easily at the plate separation stage. The gap generated by the double set of spacers does not allow for the use of standard $0.8-\mathrm{mm}$ combs for protein gel combs. This is overcome by using the Bio-Rad 14-cm plastic square combs (16 well), which are designed for sequencing. Two combs of 0.4-mm thickness are glued (or can be taped) together to form a single comb, which fits well in the gap made by the spacers. If combs are glued, a high-strength adhesive should be used so as not to increase the width significantly, and the edges of the comb are trimmed with a scalpel after gluing. New combs should be tested before pouring gels. The only other extra requirement is approximately $10 \mathrm{~cm}^{3}$ of modeling clay or plasticine, which is used for sealing the bottom of the assembled apparatus. The plasticine should be kneaded before use to make it very malleable and is split into three lots, two of $0.5 \mathrm{~cm}^{3}$ each and the remainder. The smaller pieces are pressed at the bottom corners of the assembly, over the areas where the ends of the spacers are located. Care should be taken to ensure that this area is completely dry as moisture can inhibit the sealing capacity of the plasticine plugs. 
Before pouring polyacrylamide seals, an $80-\mathrm{mL}$ stock of a sodium dodecyl sulfate (SDS)-PAGE (at whatever concentration is required) acrylamide gel solution is prepared according to standard protocols (2). The procedure we describe here was carried out with gels of approximately $13 \%$ monomer concentration and were prepared from Bio-Rad $40 \%$ acrylamide/bis solution. Gels with more than $13.5 \%$ monomer concentration tend to split during the drying step after electrophoresis. $N, N, N^{\prime}, N^{\prime}$-tetramethylethylenediamine (TEMED) is not added until required, and the amounts used should be adjusted for different gel concentrations. The plate assembly, sealed with plasticine at the bottom corners, is placed on its side. There is a $1.5-\mathrm{cm}$ protrusion at both sides of the clamps, encompassing the power connections, which gives the assembly a slight tilt while on its side. One-and-a-half milliliters of the gel stock are mixed with $2 \mu \mathrm{L}$ of TEMED and quickly introduced at the lower open corner. The apparatus can be tilted gently to allow the acrylamide to reach the plasticine plug, but it should be then leveled by placing a small block under the lowermost end of the clamp. This helps formation of an evenly distributed seal along the length of the spacers. When the acrylamide has polymerized, the apparatus is inverted and the other side is sealed in a similar manner. When this latter plug has polymerized, the remainder of the gap between the plasticine plugs is sealed with the larger piece of plasticine. The corner seals should not be removed or disturbed at these steps. This can cause leakage at later stages. The assembly is then placed in an upright position. A 5$\mathrm{mL}$ aliquot of the gel stock is added to $10 \mu \mathrm{L}$ of TEMED, and this is introduced down one side of the plate gap to form a bottom seal approximately $3 \mathrm{~cm}$ high. This latter addition should not breach the plasticine plug. If a breach does occur, then it is unlikely that the weight of the main gel will be held by the seals, and the apparatus should be rewashed, assembled and resealed. Once this last seal is successfully poured and has polymerized, the apparatus is placed gently in a vertical position on the foam gasket in the sequencing gel caster base. The screws of the caster base are tightened, but no downward pressure should be applied, as this can displace the plasticine plugs.

Before pouring the separating gel, a $30-\mu \mathrm{L}$ volume of TEMED is added to the remainder of the gel solution. The two critical problems with pouring the gel are trapped air bubbles and leakage. If there are no leaks, then $60 \mathrm{~mL}$ of gel mixture are sufficient to fill the gap between the plates. However, this volume places some pressure on the seals, and the apparatus can only be gently tilted while pouring. The base should not be moved until the gel is completely set. We have found it easier to place the apparatus at ground level for pouring. The gel is poured using a $25-\mathrm{mL}$ pipet in 20 -mL steps. The mixture is pipetted in at one or other top corners at a moderate speed since too rapid an addition results in formation of several bubbles. After each section is poured, the gel is quickly examined for bubbles. These can be dislodged with a clean 0.4-mm spacer, which has been cut to a third of its width. The full-width spacer tends to stick to the sides of the plates and is difficult to manipulate. The gel is poured to within $3 \mathrm{~cm}$ of the top of the plates. This is then quickly overlaid with 2-3 $\mathrm{mL}$ of $0.1 \% \mathrm{SDS}$, and then the gel should not be moved until it has solidified. The comb is washed and rinsed in $70 \%$ ethanol. A $10-\mathrm{mL}$ volume of $5 \%$ stacking gel is prepared according to standard procedures (2). When the main gel has set, the $0.1 \%$ SDS layer is washed out with distilled water, and the gap is dried with filter paper. The comb is placed in the gap allowing $1-1.5 \mathrm{~cm}$ from the bottom of the comb to the interface of the separating gel. The stacking gel can then be introduced at one corner by using a 1-mL pipet.

When the stacking gel has polymerized, the gel can be treated like a normal SDS-PAGE gel. Before starting electrophoresis, the combs are removed. SDS running buffer $(1.1 \mathrm{~L})$ is prepared, and $0.4 \mathrm{~L}$ is placed in the lower chamber of the sequencing cell. The remainder is poured into the inner plate chamber holding the gel. Wells are carefully flushed with a Pasteur pipet. Up to $20 \mu \mathrm{L}$ of protein sample can be added to each well generated by the 16-well comb. Electrophoresis conditions can be varied depending on the length of gel required. For a 2-3-h run (one third to one half of the plate length), the buffer should be pre-chilled and the current set at $45 \mathrm{~mA}$. For a fulllength gel, electrophoresis should be carried out overnight at a constant voltage of $400 \mathrm{~V}$. Migration of samples through the bottom half of the gel is slower. With equivalent V/cm settings,

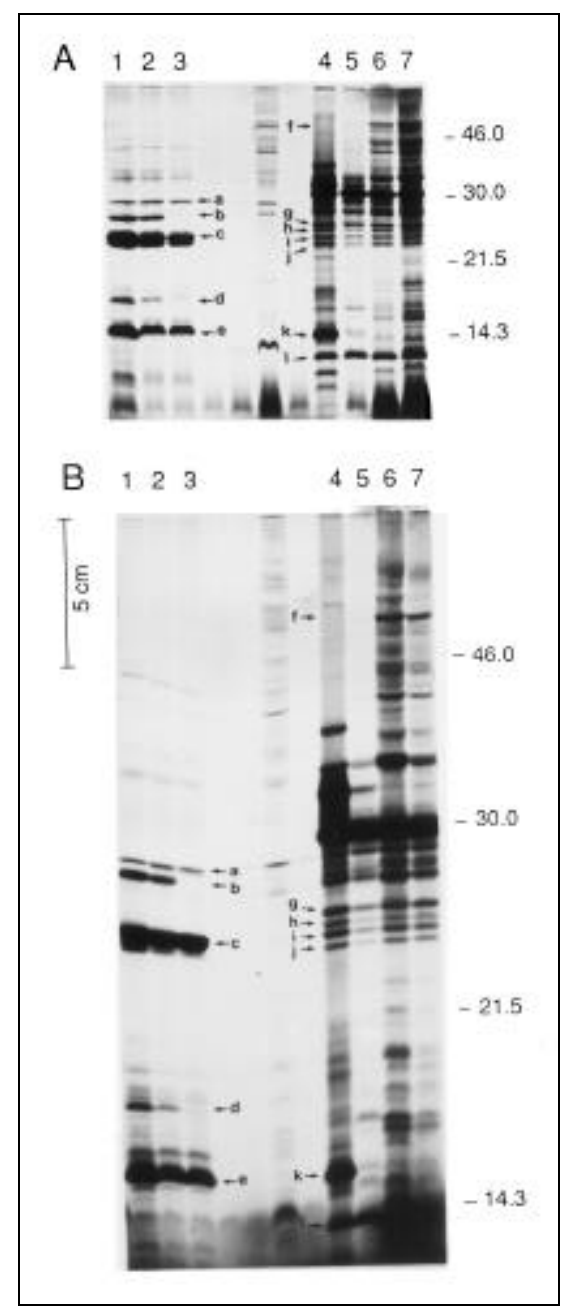

Figure 1. Comparison of SDS-PAGE of protein samples using (A) a PROTEAN II xi cell and (B) a Sequi-Gen cell. $\left[{ }^{35}\right.$ S $]$ Methionine-labeled samples were prepared using the T7 expression system $(4,6)$. Identical amounts of samples were loaded into corresponding wells for both $12.8 \%$ SDS-PAGE gels, and electrophoresis was carried out over $16 \mathrm{~h}$. Lane 1, clone pDT2765; lane 2, pDT2766; lane 3, pDT2767; and lanes 4-7, clone pDT2761. Clones pDT2665-7 contain EcoRI inserts expressing polypeptides from the plasmid R478 ter gene cluster (7). Plasmid pDT2561 contains a 24-kb $\mathrm{XbaI}$ fragment from the plasmid R27 (Whelan and Taylor, unpublished data). Molecular masses are indicated on the right in kilodaltons. Arrows indicate selected polypeptides and are designated alphabetically in both autoradiographs. 
both systems require equal amounts of time for complete runs. Then the apparatus is disassembled, and the plates are carefully prized apart. The gel is transferred to wet chromatography paper and dried using a vacuum dryer for 1.5 $\mathrm{h}$ at $60^{\circ} \mathrm{C}$. The dried gel is then examined using autoradiography.

We compared identical [ ${ }^{35}$ S $]$ methionine-labeled samples using the sequencing gel method with a Bio-Rad PROTEAN $^{\circledR}$ II xi cell (Figure 1). We found that while both systems are adequate for protein analysis, the sequencing apparatus had certain advantages. While twice as much material is required for making the gel for the sequencing system, the PROTEAN II cell requires $4.5 \mathrm{~L}$ of SDS-PAGE running buffer as opposed to $1.1 \mathrm{~L}$ for the Sequi-Gen cell. This larger volume is both cumbersome to prepare and also requires $3.6 \mathrm{~L}$ (based on a $5 \times \mathrm{SDS}$ PAGE buffer stock) of double-distilled deionized water. The PROTEAN II apparatus is initially operated at $200 \mathrm{~V}$, which generates a current of 50-60 $\mathrm{mA}$. The current is a limiting factor in the migration of proteins for this system, and higher currents cause heating of the gel and buffer. The PROTEAN II system has an inner chamber through which water can be circulated during electrophoresis to reduce heating. The sequencing system that we have used here can be used to achieve much faster equivalent-length runs as the PROTEAN II system. We have also carried out electrophoresis at $800 \mathrm{~V}$ and $50 \mathrm{~mA}$ for short 2-h runs, but we recommend pre-chilling the buffer as there is some heat generation. The sequencing system need not be used specifically for running radioactive samples. Fulllength gels makes $\mathrm{CBB}$ or silver staining very awkward, so samples should only be allowed to reach $50 \%$ of the plate length and then the gel be cut for staining. Protean II glass plates are 20 $\mathrm{cm}$ in length so that even a mid-length sequencing gel $(25 \mathrm{~cm})$ would give comparable resolution of samples. Polypeptides subjected to electrophoresis for the full length of the Sequi-Gen cell exhibit a larger degree of separation than for identical samples separated on the PROTEAN II system (Figure 1).

\section{REFERENCES}

1.Laemmli, U.K. 1970. Cleavage of structural proteins during the assembly of the head of bacteriophage T4. Nature 227:680-685.

2.Sambrook, J., E.F. Fritsch and T. Maniatis. 1989. Molecular Cloning: A Laboratory Manual, 2nd ed. Cold Spring Harbor Laboratory Press, Cold Spring Harbor, NY.

3.Sancar, A., R.P. Wharton, S. Seltzer, B.M. Kacinski, N.D. Clarke and W.D. Rupp. 1981. Identification of the $u v r A$ gene product. J. Mol. Biol. 148:45-62.

4.Studier F.W., A.H. Rosenberg, J.J. Dunn and J.W. Dubendorff. 1990. Use of T7 RNA polymerase to direct expression of cloned 
genes. Methods Enzymol. 185:60-89.

5.Switzer, R.C., C.R. Merril and S. Shifrin. 1979. A highly sensitive silver stain for detecting proteins and peptides in polyacrylamide gels. Anal. Biochem. 98:231-237.

6.Tabor, S. and C.C. Richardson. 1985. A bacteriophage T7 RNA polymerase/promoter system for controlled exclusive expression of specific genes. Proc. Natl. Acad. Sci. USA 82:1074-1078.

7.Whelan K.F., E. Colleran and D.E. Taylor. 1995. Phage inhibition, colicin resistance, and tellurite resistance are encoded by a single cluster of genes on the IncHI2 plasmid R478. J. Bacteriol. 177:5016-5027.

This study was supported by an operating grant (MT6200) from the Medical Research Council of Canada. D.E.T. is a medical scientist of the Alberta Heritage Foundation for Medical Research. Address correspondence to Diane E. Taylor, Dept. of Medical Microbiology and Immunology, 128 Medical Sciences Building, University of Alberta, Edmonton, AB T6G 2H7, Canada.

Received 23 January 1996; accepted 18 April 1996.

Kenneth F. Whelan and Diane E. Taylor

University of Alberta

Edmonton, AB, Canada

\section{Antibody Screening for Secreted Proteins Ex- pressed in Pichia pastoris}

BioTechniques 21:808-812 (November 1996)

The methyl-trophic yeast Pichia pastoris is becoming widely used as an organism for high-level protein expression (3). Commercially available vectors and host strains allow for secreted or intracellular expression of heterologous protein. When the desired end result is secretion, it is useful to have a quick and simple screening assay for secreted protein. We describe a semiquantitative colony blotting protocol for the detection of proteins secreted by $P$. pastoris growing on agar plates.

Vectors using the HIS4 gene to allow growth on media without histidine, and using the alcohol oxidase (AOX1) control sequences to allow induction by methanol, are commonly used for expression of proteins in $P$. pastoris $(3,7,8)$. Screening generally takes the form of selection on plates lacking histidine to ensure that the HIS4 gene is integrated, followed by comparative growth on minimal dextrose or methanol plates to determine the methanol utilization $(\mathrm{Mut})$ phenotype. $\mathrm{Mut}^{+}$colonies grow fast on methanol plates because the natural $A O X 1$ gene is undamaged, whereas $M u t^{\mathrm{s}}$ colonies grow slowly because the transfected gene has undergone homologous recombination into the $A O X I$ gene. Screening for integration of the gene by polymerase chain reaction (PCR) or dot blotting may also be desirable. The latter is particularly important in screening for multiple integrations, which can increase protein expression (2). Screening of colonies for secreted product is performed by growing cells in minimal glycerol medium, then concentrating them and continuing culture in minimal methanol medium. This is followed by gel electrophoresis and frequently by Western blotting. Screening a large number of colonies in this way is timeconsuming and requires substantial effort. The protocol described below requires very little hands-on time and allows screening for high-expressor secreting colonies to "piggyback" on the $\mathrm{Mut}^{+} / \mathrm{Mut} t^{\mathrm{s}}$ comparative screening, thus bypassing screening for high-copynumber integrants by DNA dot blotting.

After transfection and selection for incorporation of the DNA on regeneration dextrose base (RDB) agar plates and/or G418 plates [i.e., for $\mathrm{His}^{+}$phenotype and for multicopy integration, respectively (6)], colonies are picked and touched onto minimal methanol (MM) and minimal dextrose (MD) plates sequentially. After incubation at $30^{\circ} \mathrm{C}$ for $1-2$ days, large colonies are present on the MD plates. The MM plates have either small $\left(M u t^{\mathrm{s}}\right)$ or large $\left(\mathrm{Mut}^{+}\right)$colonies. At this point, the colonies are scored for the Mut phenotype, and the MD plates are stored at $4^{\circ} \mathrm{C}$. A nitrocellulose filter (Schleicher \& Schuell, Keene, NH, USA) is placed over the colonies on the MM plate, followed by three sheets of 3MM chromatography paper (Whatman, Maidstone, Kent, England, UK). Several towels are added on top, and the pile is weighted down with about $50 \mathrm{~g}$. The plate is incubated at $30^{\circ} \mathrm{C}$ for $1-3 \mathrm{~h}$. Omitting the blotting step, simply laying the nitrocellulose filter on top of the colonies is less sensitive, which can be an advantage in selecting high-secreting colonies (Figure 2). The nitrocellulose filter is removed and washed in tris-buffered saline (TBS: $20 \mathrm{mM}$ Tris base, $137 \mathrm{mM} \mathrm{NaCl}, \mathrm{pH}$ 7.6) to remove the yeast colonies. The next steps are identical to standard Western blotting protocols and require specific antibody reagents for the particular proteins under investigation (see below). High-expressing colonies identified in this manner are recovered from the MD plate and grown up for more specific assays of protein expression.

In the example presented here, we transfected $P$. pastoris strain GS115 with a gene for the $\mathrm{T}$ cell receptor (TCR) $\alpha$-chain cloned into pPIC9 (6) (Invitrogen, San Diego, CA, USA). The TCR $\alpha$-chain gene was truncated to allow secretion $(4,5)$. The cells were transfected by electroporation and plated onto RDB plates to select for incorporation of the HIS4 gene. We tested colonies growing on RDB for incorporation of the transfected gene by standard techniques; MM vs. MD plates for Mut phenotype, followed by DNA dot blotting for $\alpha$-chain incorporation. For this demonstration experi- 